\title{
Multicast Routing Protocols for Mobile ADHOC Networks
}

\author{
V.Sabaresan , S.Godfrey Winster
}

\begin{abstract}
Mobile Ad-hoc Networks (MANETs) is a selfconfiguring network that doesn't follows any predetermined infrastructure. In MANETs there are collections of independent nodes which are associated through wireless and at the same instance it is allowed to change its links to other nodes frequently without any fixed infrastructure. Multicast routing is concurrently sending a message from single source to several destinations and it is an efficient approach to establish the communication between groups of nodes in the wireless network. Multicast routing plays very important roles in the fields of video conferencing, co-operative works, distance education, video on demand, cyber database updating and querying, etc. These applications can provide themselves to manage multicast operations. Almost all different type of multicast routing algorithms in MANETs are facing various issues like limited bandwidth, possibility packets loose, delay variance, energy consumption and transmission overhead problem. There are several multicast routing protocol has been proposed to overcome the challenges of multicast routing and play very significant roles in the current scenario. Nowadays, dissimilar multicast routing algorithms with unique characteristic have been introduced. In this paper complete survey on multicast routing algorithm to highlight their advantages, limitation and also show the future direction in multicast routing in MANETs.
\end{abstract}

Keywords: MANETs, Wireless networks, Routing and multicast routing protocol

\section{INTRODUCTION}

Wireless network is a special kind of data communication networks which uses wireless connection omits the costly technique of the installation of cable for the data connection among devices with different location. Wireless networks are basically separated into two types namely infrastructure and infrastructure-less networks.

Revised Manuscript Received on October 12, 2019

V.Sabaresan , Assistant Professor, Department of computer science, Kalaivani College of Technology,

S.Godfrey Winster, Department Computer Science and Engineering, Saveetha Engineering College. mechanisms for exchanging information among nodes. It

MANETs are dynamic, multi-hop, self- constituting and self-organizing, wireless adhoc networks. It contains huge number of nodes in a network and not requires any type of fixed infrastructure. In MANETs nodes are works as a router which find and maintain the path from source to destination. Topology in the MANETs can be changed dynamically due to the mobility of nodes [1]-[3]. In the environment of MANETs there are two most important issues to break the communication between nodes while the data transmission in a network. First reason is nodes die due to the energy exhaustion because mobile nodes have very small amount battery capacity and second one since the nodes are moving out of range from its nearest nodes. [4] [5].

MANETs are deployed and implemented in various areas using different ways and plays an significant roles in several application such as disaster area recovery supports, commercial sectors, individual spontaneous networks, data and device networks [6]-[10]. MANETs are varied series of challenges and proceeds in the field of dynamic topology, quality of service [QOS], energy constrained operation, device discovery, security, reliability, limited device and bandwidth constrains [10][20]. Routing is a process of finding best suitable paths in networks at a particular situation and it is responsible for forward packets in the network and host may be sources and sinks [21]. Route traffic between nodes is a huge challenge to overcome as there are many and frequent communication among nodes and this leads to packet distribution problems in very huge networks [22]. The paper is organised as follows in chapter II, various routing protocols for MANETS are discussed. The basic investigation on characteristics of multicasting routing algorithm is elaborated in chapter III. In the chapter IV the classification of techniques on multicasting routing protocol is analyzed and reviewed. In chapter V conclude the review by presenting an outline of the routing strategies that are depended on their characteristics and show the direction for the future works regarding multicast routing algorithm for MANETs.

Published By: 


\section{ROUTING PROTOCOLS FOR MANETS}

There are various routing protocols can be proposed on MANETs and divided into subsequent types based on their routing methods namely unicast, Geocast, Anycast, Broadcast and Multicast routing protocols.

\section{A. Unicast routing protocols}

Unicast routing protocol is a very basic mechanism in Mobile Adhoc Networks to find the optimal path for transmit data from source to destination nodes [23]. The figure 1 demonstrates the process of unicast routing protocols. In unicast routing transmit packets from one source to a single already specified destination. Forwarding process is extremely trouble free to establish a connection between sources to destination nodes through routing table. A communicating node, directly access and handle target address inside packets with in a routing table.

If most feasible corresponding target address is established in a connection depending upon the need of the application. Unicast routing protocols classified based on their network structure and routing strategy. We classify these routing protocols into following type's namely proactive, reactive, hybrid and hierarchical unicast routing algorithms for wireless Ad-hoc environments [24].

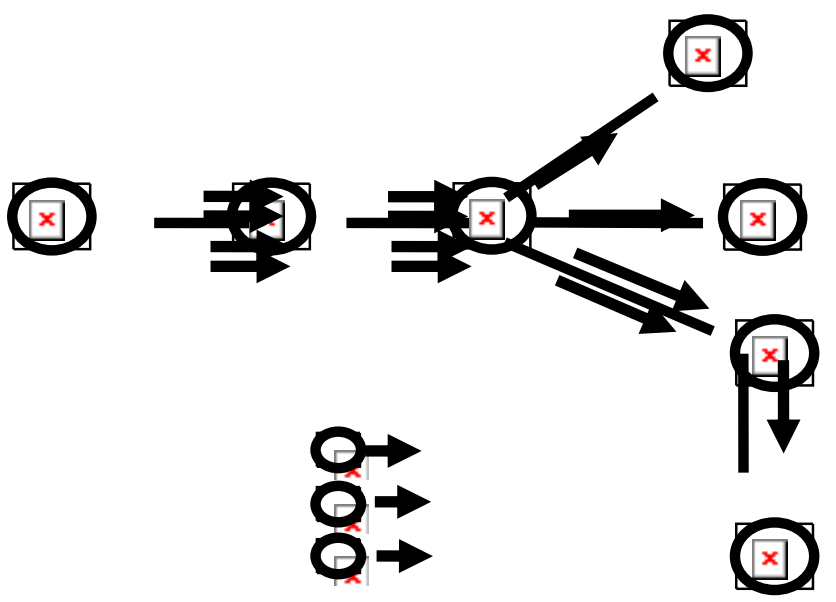

FIGURE 1: Data packet is replicated to the sender

\section{Proactive unicast routing protocol:}

It is more over known as table driven routing methodologies because, they retain the routing information before routing has starts. Each node in a network requires to maintain routing information about other nodes and that information is usually retained inside the routing table in addition to it was regularly modified as based on their structure in a network [25]. Limitations of these routing protocols are high resource utilization and significant of overhead problem. Following the some example of proactive unicast routing algorithms; there are Wireless Routing Protocol (WRP), Fisheye State Routing (FSR), and Optimized Link State Routing Protocols (OLSR) [26].
Reactive unicast routing protocol is a demand based routing algorithm, since they are no need to maintain the routing information when no transmission occurs in a networks. If a node need to send a packets to other nodes in a network then this protocols find and establishes a path in according to their query packets as on demand method. The different type of reactive unicast routing algorithms are TORA (Temporally Ordered Routing Algorithm), AODV (Ad-Hoc on Demand Distance Vector), DSR (Dynamic Source Routing), and ABR (Associatively Based Routing) [27].

B. Hierarchical unicast routing protocol:

In hierarchical unicast routing protocol nodes are separated into several cluster, each group contains cluster heads and number of cluster nodes [28]. It is an effective and scalable routing mechanism by increasing their size beyond a certain threshold in a hierarchical manner.

It is a key suggestion to the nodes in different groups and defining function to nodes in a network [29]. TEEN (Threshold Sensitive Energy Efficient sensor Networks), APTEEN (A Hybrid Protocol for Energy Efficient Network), PEGASIS (Power Efficient Gathering in Sensor Information System), LEACH (Low Energy Adaptive Clustering Hierarchy), and TTDD (Two-Tier Data Dissemination) are example of hierarchical unicast routing protocol [30].

\section{Hybrid unicast routing protocol:}

Hybrid unicast routing protocols are combination reactive and proactive routing algorithms in addition to overcome the limitation of reactive and proactive routing protocols [31]. It uses route discovery approach from reactive routing protocols and table maintenance approach from proactive routing protocols, thereby it reduces the latency, overhead problems and it is appropriate for large group of nodes with provide high scalability in a network than pure reactive and proactive routing algorithms [32]. The ZRP (Zone Routing Protocol), ZHLS (Zone Based Hierarchical Link State Routing) and HARP (Hybrid Adhoc Routing Protocol) are example of Hybrid unicast routing protocols.

\section{Observation on Unicast Routing protocols:}

Unicast routing algorithm is a simplest operation to transmit packets from single source to already specified destination. Route discovery is main process of the unicast routing algorithm and it controls nodes decisions in MANETs. Unicast routing algorithms are classified into four categories, they are proactive, reactive, hierarchical, and hybrid unicast routing algorithms. Proactive unicast routing algorithms are commonly known as table driven routing algorithm as they

Reactive unicast routing protocol:

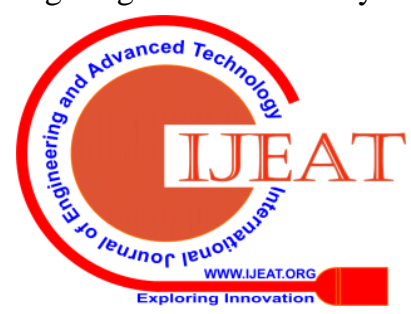


frequently update information in routing in routing table.

\section{E. Geocast routing protocols}

It is basically a location based mechanism and send collection of packets across a wireless networks to others simultaneously in a specific geographic region. In Adhoc networks Geo-cast routing algorithm does not maintain exchange information between neighbouring nodes [33]. Classification of Geo-cast routing algorithm can be done in numerous different ways, but most of them are completed depending on whether they are designed for infrastructure and their geographic destination. According to the Ad-hoc and infrastructure less are classified as flooding, directed flooding and no flooding. While depending on fixed infrastructure networks are classified as no flooding. Figure 2 shows the classification of Geocast routing algorithm [34].

\section{Observation on Geo-cast Routing protocols:}

Geocast routing is one of the important and most persistent communication services where it transmits to nodes within a specific geographic area. Whatever it may be the Geocast target region, the simple flooding spreads the entire

Deterministic broadcast routing protocol is a collection of rely nodes, there itself chose decision and resolve to cover the entire networks. It is also guarantees a full coverage of networks in very efficient way. Deterministic broadcast routing protocol depends on BFS (Breath First Search) traversal to find optimal path in networks and allows communication between multiple nodes simultaneously at a time [43]-[45]. In simple flooding approach, a source node transmits packets to every neighbouring node in the whole network and all intermediate nodes that check whether the message is previously received or not. If message is received already, then it will be relinquish or else the message will be retransmit till the intermediate node receives the message. The main intention of probabilistic routing protocol is used to minimize the utilization of network properties like bandwidth and energy to avoid rebroadcasting the same message repeatedly [46]-[48].

\section{Observation on Broadcast Routing Protocols:}

In multicast routing protocol a node can send packets to all of its neighbours and multiple destinations simultaneously with their networking range. Probabilistic broadcasting routing protocol is best suitable for its decentralized network nature and it also helps to control the dissemination of the routing control packets. Probabilistic approach is most feasible ways to minimize rebroadcast. Deterministic broadcast protocol that do not require any type of routing information in routing table in order to

information network topology in addition to they can operate and maintaining broadcast spanning tree. It is advance determination of the route between single sources to more than one destination. In static routing protocol, the table can altered and modified manually. It can be more efficient in only small networks and very difficult to reconfiguration. In dynamic routing protocol the table can build and altered automatically with help of routing protocols. Dynamic routing is used to overcome the limitation of static routing mechanism.

\section{F.Multicast routing protocols}

Multicasting is a process of a source node sending several packets of data to single or multiple destinations simultaneously mean while delivery of single message over the entire link in the network only once and create several message when the link is splits into more than one destination [49]. It allows to access by wired networks, infrastructure based wireless networks, and applying this mechanism to mobile adhoc network is a big challenge to overcome [50]. Multicasting is a very significant mechanism in which it makes very easy to access lot of real time applications and allows group of communication as a replacement for exchange information between two individual nodes. The most important objective of multicasting routing algorithm is to accurately distribute packets to multiple nodes in a network [51]. In multicast routing algorithm generally uses two type of models; there are, one to one model and many to many model. In earlier we can have at least one sender and multiple receivers in the network. Figure 4 illustrate the process of one to one model in broadcast routing protocol [52].

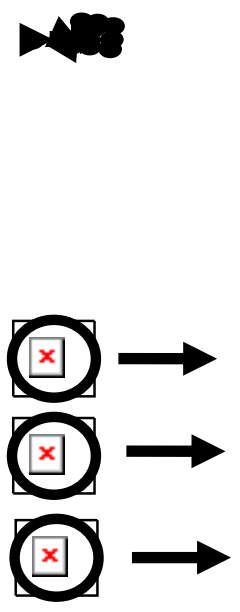

FIGURE 4: Process of one to one model

In many to many models, it can have many sender and multiple receivers in the network. Figure 5 displays the process of many to many model in broadcast routing protocol [52].

Published By. 

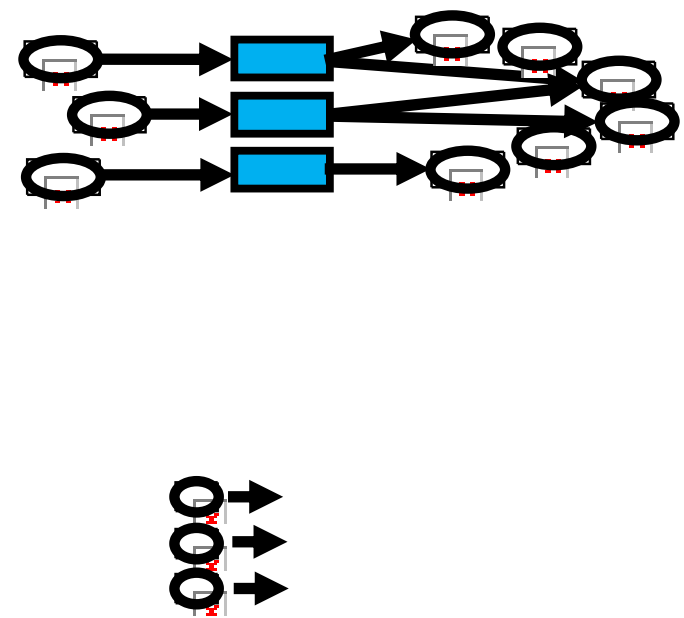

FIGURE 5: Process of many to many model

\section{Observation on Multicast Routing Protocols:}

Multicast routing protocol plays a very essential responsibility in adhoc networks to send packets from origin to several destinations in a point of time. The most important problem is to overcome a multicast routing protocol in MANETs is to manage the frequently changing topology characteristics in a network. It can improve the networks performance by optimizing the parameters such as bandwidth, Quality of service (QOS), through put nature and channel utilizations [53] [54]. Topology change plays key role in designing an optimized multicast routing protocol. In designing they depend upon topology discovery for finding optimal path when nodes movement are extremely high in the MANETs. However a source node is possible to transmit data to alternative nodes in the transmission range [55].

\section{CHARACTERISTICS OF MULTICASTING ROUTING PROTOCOL}

They are various characteristics, parameters and limitations that should be measured at what time we need to implements and design the multicast routing procedures in MANETs such as restriction of limited battery power, availability of bandwidth, unpredictable structure, poor efficiency, lack of scalability and reliability, low robustness, depends upon unicast routing protocol and security problem as discussed below [50], [56]-[60]:

Reliability: In MANETs military and other difference type of real time applications is not tolerable to drop any packets during the transmission and must receive entire packets perfectly from origin to destination, as a result multicast routing protocol must suppose to be guaranteeing this attribute.

Battery power Consumption: In the network, the failure of power limitation of source systems causes the node failure, thus by minimizing the end to end connectivity. The regular and repeated transmission of nodes which determines the life duration of nodes in the network. Now a day's some of proposed multicast routing protocol within MANETs mainly focus on the approaches to optimize routes which are mostly based on the shortest path and smaller amount of energy at the node level which increases end to end communication among nodes in the network and reduces the fault at the node level [61], [62].

Efficiency: The capability and ability of multicast routing protocol depends on the ratio between entire packets arrived by destination nodes and total numbers of packets send by source nodes within a network. Temporary and wireless adhoc networks can be constructed using the multicast routing protocols and it plays a significant part. Where the nodes can be move erratically, routing protocols are highly effective and assure successful packet delivery. In networks where the bandwidth is restricted the multicast routing protocols in MANETs can be used significantly [63].

Robustness: Now a day's, MANETs has gained attention and one of the effective methods for group communications because of group membership management and dynamic topology is difficult and it is very powerful and strengthens multicast approach. Due to the breakage of link some data packets in MANETs are lost. When the node travel away from the broadcasting area, then breakage of links may occur and which will leads to low packet delivery ratio. High PDR that have high movement of nodes can be attained by strength of multicast routing algorithm [64].

Scalability: In the network sources, destination and intermediate node can be handled and controlled using the multicast routing protocol and this ability is called as scalability. As the increase of nodes in the network during session then it leads to more control packets can involved in the multicast routing protocol. Since there is a motion of nodes and constant change in the topology it is inconvenient to design a consistent and ascendable routing protocol for MANETs.

Bandwidth Utilization: In MANETs multicast routing protocol minimize bandwidth consumption and transmission cost. Group communication may be used, even though it has huge number of participation in its communication rather than various unicast routing protocol.

Security: Security is also a more challenging part because the open access is additionally exposed to external attacks and the multi-hop communications make the process more risks to internal attacks coming from comprises nodes. These attacks are not only having a straight effect on the multicast communication, but also raise further participation to protocol the multicast session from any illegal operation [67], [68].

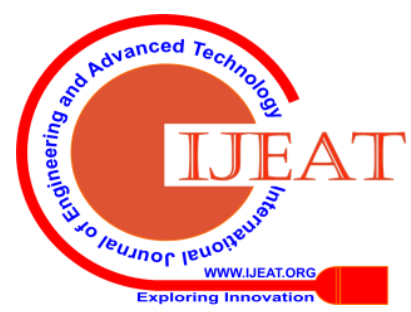


Need of unicast routing protocol: Now a days, various proposed protocols are available for several purpose with dissimilar properties. These networks are named as disparate network and occasionally multicast routing protocols are necessary to work together with unicast routing protocol.

\section{CLASSIFICATION OF TECHNIQUES ON MULTICAST ROUTING PROTOCOL}

Taxonomy of techniques on multicast routing protocol can be prepared in several different ways according to their structure; it can be categorized as tree, mesh and hybrid supported multicast routing protocol. Figure 6 illustrate classification of multicasting routing protocol.

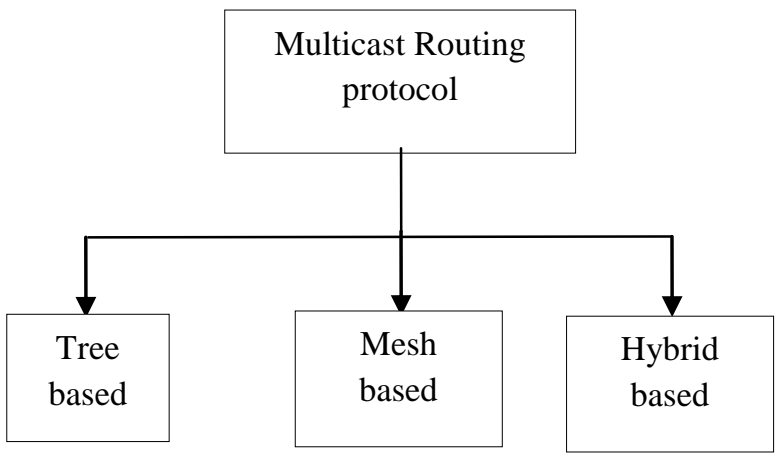

FIGURE 6: Taxonomy of multicast routing protocol

\section{A. Tree based multicast routing protocol:}

In tree based multicast routing protocols, all the routers obviously appear in the form of tree structure by means of the source node at the same instance as root node and there is exactly single path between each group of source and receiver, as the result keep away from redundant transmission. Tree supported multicast routing protocols are afforded link transmission with good organization at the cost of strengths and it provides very efficient tree reconstructions while network failures [69], [70].The structure of tree supported multicast routing protocols can be frequently changeable and unstable, so it needs to be a regular rearrangement of nodes in dynamic networks. Even though tree based approaches in MANETs bandwidth is very efficient, it always provides sufficient strength during risk in movement of nodes or high mobility and link failures [71]. Tree based multicast routing algorithm might be moreover sub divided in several ways according to their network head of multicast tree. It might be categorized as source rooted tree depended multicast routing algorithm and core rooted tree depended multicast routing protocol. While depending on their tree structures these classified as source tree depended multicast routing protocol and shared tree routing protocol. Figure 7 illustrated sub division of Tree depended multicasting routing protocol. [72] - [74].

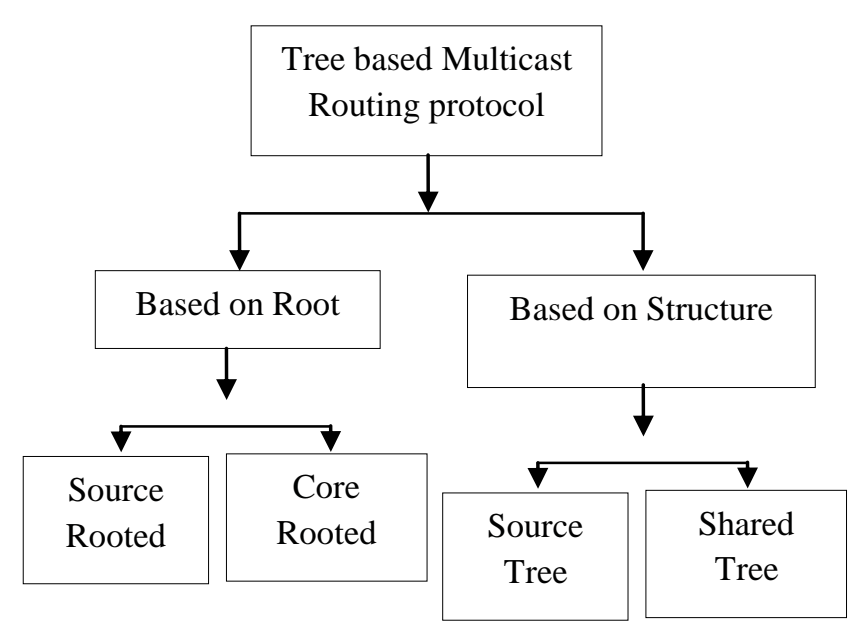

FIGURE 7: Classification of Tree based multicasting routing protocol

\section{B. Source Rooted Tree based multicast routing protocol:}

Source Rooted Tree depended multicast routing protocols uses source node as a root node of multicast tree in addition to perform algorithm for allocation tree structure and maintenance. In these protocols source have to be responsible of handling topology information along with address of each and every receiver in the multicast cluster. Whenever the dynamic networks are used, source rooted tree based routing protocols sustain from high traffic over head problems.

\section{Core Rooted Tree based multicast routing protocol:}

In core rooted tree based multicast routing protocol; root treated as a node with significant purposes like considering that multicast information sharing and link organization. Several core rooted tree depended approaches and make use of tree structures but not like source rooted tree depended algorithm, in case of multicast tree rooted node is only considered as a core node, unlike source rooted routing algorithms, core or root nodes can perform a variety of routing and management operations. It provides high data forwarding effectiveness at the rate of low strength. Their advantages are simplicity and tree structure is reconstructed in very high mobility until when packets are possibly had to drop.

\section{Source Tree based multicast routing protocol:}

For the period of this source tree depended multicast routing protocol, each and every source node constructs a particular multicast tree, which consists of entire connecting nodes within the network group. In general, single path among the source and each other node in the network, which may not be a shortest one compare with other paths.

\section{E. Shared Tree based multicast routing protocol:}

During this approach a corresponding multicast group surrounding on each and every

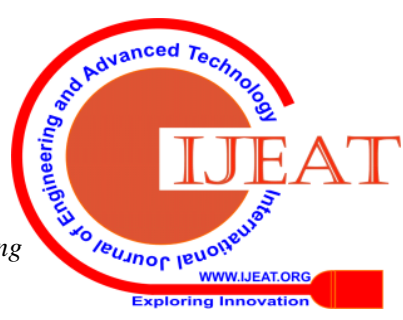




\section{Multicast Routing Protocols for Mobile ADHOC Networks}

source node is produced for MANETs.

After that, root node as a core node and every source node make use of this tree to establish multicast routing. This shared tree based algorithms are suggested for shortest path for routing, on other hand it treats particular point of failure, and so it frequently perverse more routing information which is directed to overhead problems. By means of this, the traffic is combined then it provides low throughput. In MANETs, various different tree based multicasting routing algorithms have been proposed, so it not possible to cover all protocol in this survey paper, discussed in details about dissimilar features, characteristics based on their performance of these routing approaches.

\section{F. Multicast Adhoc on demand Distance Vector (MAODV)}

In MANET, the protocol permits to organize themselves and dynamic multicast routing between groups of mobile nodes that are required to add or contribute in the multicast group. The lifespan of the network is related with their multicast group members. Multicast tree establish a connection among their mobile nodes using this approach. Without any kind of time delay the mobile nodes will reply rapidly to link disruption in the above said multicast trees by fixing these interferences. By using the broadcast route discovery methodology the MAODV routing protocol finds the demands in the multicast routes. Even if the mobile node doesn't have any connection to a group, it creates a Route Request (RREQ) communication once it desires to link a multicast cluster or as soon as the data has to be forward to the multicast cluster. Route Request (RREQ) will rely only to the chosen element of the multicast group. In the multicast cluster, if the Route Request (RREQ) doesn't have a request, latest routes in this multicast cluster can be relied. If the in-between nodes accepts a link Route Request (RREQ) in the multicast group that is not participated, or it obtains a Route Request (RREQ) that doesn't have any link to that group, so the RREQ rebroadcasts to its nearby groups. For instance if the RREQ is transmitted across the network the nodes in the mobile network sets up pointers which begin to retract route in the route table. [78]-[81].

\section{G. Differential Destination Multicast Protocol (DDM)}

Differential Destination Multicast protocol proposed in the company for more than limited goals. The choice of the deported multicasting is provided by this DDM protocol. The option of managing and promoting reversed state and demanding its challenging to neighbour, then subsequently including this condition into self-route data packets or a number of combinations thereof is done by each and every node independently. The main goal of this DDM protocol is that it most suitable for dynamic networks with huge number of nodes and utilizes multiple channel access with very cost mechanism compared to other proposed protocols in tree based routing protocol.

The next purpose is to introduce and design a protocol, which permits centralized group of node organization. The next goal of this protocol is more flexible in addition to providing alternative configuration space for each nodes with different parameters according to their behaviour in any local environment [82], [83].

\section{H. Location Guided tree construction protocol (LGT)}

The small group of protocol depends on packet enclosed technology and it is known as LGT (Local Tree Construction Algorithm). In multicast data, this holds a destination list to be encapsulated inside the unicast packet. The LGS (Local - Guided Steiner) and LGK (LocationGuided K-array) protocols for packet, forward methodology is used by which nodes positioned in the destination list inside packet. By using the fusion mechanism this procedure improves the tree structure via the router and frequently update their position of nodes. By considering the limitations such as bandwidth and control overhead, the tree construction algorithm of LGT chooses the best feasible route. The source initiated shared tree algorithm and hard state protocol approach is owned in LGT. In this LGT, protocol doesn't include any topology, packet sharing approaches and it mostly considers the geographical information of the node. The network structure and network distance are calculated by this protocol when a node is distant from the network hops. In Local Tree Construction Algorithm only grouping nodes are treated as tree nodes. By contrast with previously produced tree algorithm locates and finds the most recent paths. Since these algorithms are used to retain global state information that is helpful within limited nodes in a network. These algorithms are used to compute in an extremely active mobility environment even though it has lot of drawbacks such as low bandwidth utilization [86].

\section{Bandwidth Efficient Multicast Routing Protocol (BEMRP)}

$\mathrm{ABC}$ optimization technique with revere path finding approach is applied in the proposed system (BEMRP) and discovers multiple routes, when it requires. Route Discover, Route Updating and Route Maintenance are the stages of the proposed BEMRP protocol. The bandwidth competencies is important intend criteria for multicast protocol, because that Adhoc network functions in high bandwidth utilization and very dynamic environment. This approach locates the adjacent redirecting node relatively shortest path and hard state multicast tree approach is used to maintain furthermore minimizing the amount of packet transmission. Once the link breaks, node spread the needed control packets to respond the multicast group \& tries to find the adjacent forwarding node. The route optimization is done to remove the unwanted forwarding nodes and thus the bandwidth usage is

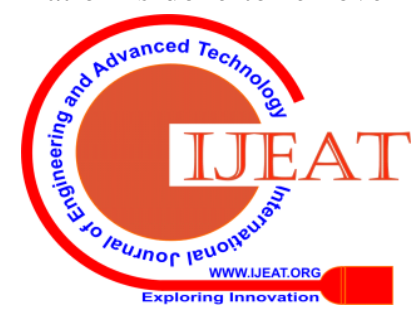


reduced. As it need minimum transmission overhead and doesn't need a cyclic broadcast of control packets. If the link breaks, then the node needs to transmit control packets [87][89].

\section{Adhoc Multicast Routing Protocol utilizing increasing id-numberS (AMRIS)}

AMRIS is tree based proactive protocol. Tree initialization and maintenance are the two major mechanisms in AMRIS. In the tree initialization method where a multicast session is generated and proclaim to nodes inside ad hoc network. Nodes that wish to join in multicast session and then to join in initialization phase are recognised as I-nodes and the nodes which are not desired to join are called as U-nodes. Inside the distribution 2 of 5 trees headfirst to multicast traffic, these U-nodes can yet become the part of this multicast session when it is essential for them to act as "midway" nodes. Tree maintenance is the technique where the nodes which becomes separated from the multicast dissemination. By this maintenance approach re-enter the tree to keep on getting multicast traffic by implementing Branch Reconstruction (BR) Routine and the nodes that doesn't wishes to join this multicast session at the time of initialization phase make hold of BR to add the tree. AMRIS practise soft state ideal methodology to detect the link that has been broken among adjacent nodes. The major aim of AMRIS is every node is identified using multicast session member. Source identified with this multicast session member is known as session ID and transmit new message with multicast session member [97], [98].

\section{B. Mesh based multicast routing protocol:}

In this method the source node to every destination nodes in the networks can be able to communicate based on the mesh topology. This approach provides excellent in terms of removing link failure in the conditions of high packet delivery rate because it offers multiple path connection between source and any other connected nodes. However this multicast routing protocol is suffering from lot of issues like power deficiency, capacity wastage, loses of data packet delivery and it is shows the way to further over head problems. Anyway mesh depended multicast routing protocol is advisable for wireless adhoc networks then tree depended multicast routing protocol [75]-[77].

\section{Core Assisted Multicast Routing Protocol (CAMP)}

CAMP is initiated through a receiver and it functions based on the demand. To initiate and manage multicast group members to destination by mesh based topology. The network individually forward loops free packet delivery and maintained by multicast meshes. For information sharing within each and every multicast group is established and retained by multicast mesh where CAMP to be varied from most previous multicast routing protocols. Within the multicast group which has at least single pathway from all sources in the direction of each receiver which is being provided by the network structure and it is a division of multicast mesh.

The direct paths from receivers to sources are branch of group mesh. From the beginning which node reaches at the router from the sources are being promoted by the multicast mesh meanwhile, shortcut from the source to receiver to be defined. It initiates a substantial number of overhead, unpredictability and irregularity in huge amount of nodes in the networks. All the mechanisms of the tree and its interconnected nodes must be rejoined for packet forwarding when the network fails and it will carry on the transmission between the origin node and the target node [90], [91].

\section{Dynamic Core Based Multicast Routing Protocol (DCMP)}

DCMP is established by source node and it is scalable, efficient in addition to extremely adaptive multicast routing protocol. The amount of forwarding nodes are decreased where the amount of source nodes are minimized which floods JoinReq packets in the Dynamic core based multicast routing protocol. The active, reactive and coreactive bases are the three groups of source in DCMP. When the source node has to send data, then JoinReq directing packets are floods in the DCMP protocol. The core acceptance flag is the additional flag of the JoinReq. Due to the MaxPassSize factor constraint core node possibly able to support additional reactive sources. If it cannot maintain further more inactive source nodes after that the CoreAcceptance flag is reset in the JoinReq packet. The nearby node will find whether this core node can support or not by checking this flag. The request to the nearby source nodes to the core is being prevented by unwarranted requests [92], [93].

\section{On-Demand Multicast Routing Protocol (ODMRP)}

This protocol depends on mesh based routing protocol along with the on demand protocol rather than a usual tree based multicast method and forwarding group is used to communicate the mesh. To locate the constant path finding in ODMRP is the vital goal of this algorithm for forwarding packets. The minimum amount of interruption principle was used in on- request routing algorithm to find optimum path for wireless Adhoc networks. To construct the path and maintain multicast group of mobile nodes actively based on demand approach. The mobile nodes bandwidth is limited, network structures can be changed on a consistent basis, and power utilization is controlled by ODMRP. It depends on interruption characteristics of connecting nodes and get better multicasting algorithm

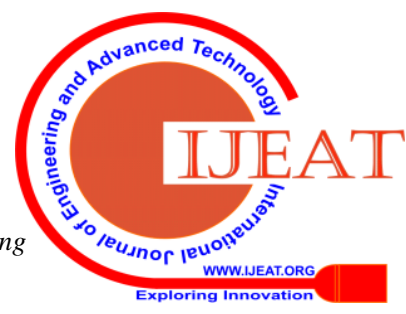


by expertly organizing flood method. The sources on demand then it can establish and update the group member using multicast routes in ODMRP. A request stage and reply segment is attached within this protocol, which is comparable to unicast routing approaches. It frequently transmits to the whole network with associated supporting packet, even though a source node has to send packets in addition to update information route and connection information by this periodic transmission [94], [95].

\section{Hybrid based multicast routing protocol:}

At the cost of less robustness, the tree based protocols achieves maximum efficiency in data redirecting. The simplicity is one of their main advantages. The disadvantage of this protocol is rebuilt their structure every succeeding motion of node, the packets probably have to drop. During forwarding the data packets, the mesh based protocols execute enhanced high mobility circumstances as they offer dispensable path from origin to destination. The mesh based procedures discard multicast effectiveness in contrast to the tree based mechanism and gradually there is an increase in the network load. This hybrid multicast routing algorithm combine the strength of tree and mesh based methods, i.e., the quality of mesh based multicast routing algorithm and minimum overhead of tree based protocols. Therefore the hybrid protocols review both the effectiveness and quality. By this, more routing path and identical message can be outstretched to the receiver over many paths [84], [85].

\section{Reliable and Energy Efficient Hybrid Multicast Routing Protocol (REHMRP)}

This protocol is a combination tree and mesh based multicast routing procedure for MANETs, Which is managed by following stages. Division of node continues with more power level by implementing energy based designed model. Directing the node dependability critical issues based on energy extent of a node, communication strength and mobility. For reducing the neighbouring nodes with the purpose of reliability critical issues and remaining power not as much of predefined entry value. Detection of multipath multicast routes that are utilizing route request and responding packets. Selection of feasible pathway from the mesh for data communication relies on path dependability importance feature and route continuation beside with negative dependability issue. Route protection is necessary in occurrence of connection breakdown, node disappointment and misplacement of Radio frequency. Disappointment of node and connection is considerably minimum in this protocol since these methods construct the dependable and constant path from origin to target node by taking into additional consideration of reliable and power capable node but that is unusual to organize the network difficulties [101].

\section{Power Controlled hybrid Multicast Routing protocol (PCHMR)}

This protocol is a hybrid multicast routing algorithm for MANETs, which collectively contains tree and mesh based construction. PCHMR protocols do not depend on hop counts and it is based on the established energy strength of the nearest nodes by route determination method.

In majority of the previous multicast routing procedures, the path selection depends on the least hop counts among the Mobile Node (MN) consideration and it is suitably designed for the dynamically altering network structures, particularly in which nodes are frequently moving in the network. The mobile node with the purpose initially creates the construction, that is grouping of mesh and tree is called the association header. The association header is also called as group header. Furthermore it is responsible for protecting the combination of construction by frequently distributing the Creation - Hello messages to entire nodes in the network. The configuration cycle number is also well-organized to be familiar with the uniqueness of the data from the group header [100].

\section{Adhoc Multicast Routing Protocol (AMRoute)}

This protocol is under the categories of hybrid multicast routing procedure for MANETs, which is designed for strong IP Multicast by making use of usermulticast trees and active cores in wireless adhoc networks. It initiates a shared tree approach for data transmission by the group receivers and senders as a node in network. Unicast channels are used to communicate nearby nodes through bidirectional way. This routing protocol doesn't necessary to be maintained by network, a node that is not aware and need of multicast, where the overhead is acquired only by group senders and receivers. This algorithm creates self assured nodes "core nodes" to establish the indicating component of AMRoute, which detect grouping members and tree arrangement. Core nodes are different from those tree supported multicast routing structural design and autonomous multicast sparse method, because they are not an inner position for data sharing and are competent of moving around actively between member nodes. AMRoute is self-determining particular unicast routing method as a result, it can function immaculately greater than unconnected field with dissimilar unicast protocols [99]

Enhanced On Demand Multicast Routing Protocol (EODMRP)

In the E-ODMRP the forwarding nodes has a limited

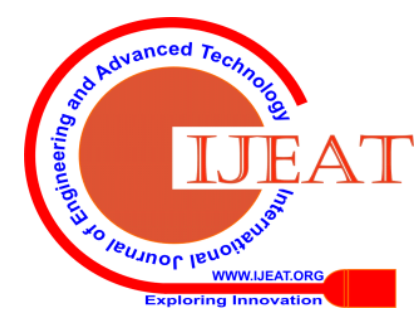


life span, but in the ODMRP the forwarding nodes has a relaxation, which will refresh 3 times in a constraint interval. The forwarder nodes free themselves by the time the forwarding situations ends by a break. In E-ODMRP the midway nodes to the main data packets provided that a receiver survives or else they end themselves [96].

\section{CONCLUSION}

Multicast routing protocol can competently support an extensive range of applications that are categorized by secure quantity of association that are many characteristics MANETs. The design of multicast routing procedure mainly motivated by particular objectives in addition to necessities that depends on the basis about the network properties and application areas. The most important objective of this research work is to evaluate the multicast routing procedure and to analysis the initial routing selection fundamentals. The protocols that are chosen from the class of identical approaches are discussed based on their features, routing metrics and principles that are reflected in this research work on multicast routing procedure. The grouping of the initial routing concepts can abbreviate the mission of a network designer in determining the multicast routing approaches to be assumed at given condition. In this paper Table represent the summary of tree depended multicast routing protocol with their behaviours. Whereas the detailed review of the mesh based routing procedure is briefly explained in Table 2. And in Table 3 the characteristics of the hybrid multicast routing protocol is considered This paper permits the researchers to have a preliminary idea about the MANETS. In any case, there are yet to be focusing many areas on further examination.

\begin{tabular}{|c|c|c|c|c|c|c|c|c|c|c|c|}
\hline $\begin{array}{l}\text { Prot } \\
\text { ocol } \\
\text { Na } \\
\text { me }\end{array}$ & $\mathbf{R S}$ & IA & $\begin{array}{l}\mathbf{M} \\
\mathbf{A}\end{array}$ & $\begin{array}{l}\mathbf{E} \\
\mathbf{A}\end{array}$ & $\begin{array}{l}\mathbf{U} \\
\mathbf{S}\end{array}$ & $\begin{array}{l}\text { M } \\
\mathbf{C} \\
\mathbf{O}\end{array}$ & $\begin{array}{l}\mathbf{P} \\
\mathbf{C} \\
\mathbf{M}\end{array}$ & $\begin{array}{l}\mathbf{L} \\
\mathbf{F}\end{array}$ & $\begin{array}{l}\mathbf{O} \\
\mathbf{S}\end{array}$ & $\begin{array}{l}\mathbf{C} \\
\mathbf{P} \\
\mathbf{F}\end{array}$ & $\begin{array}{c}\mathbf{A} \\
\mathbf{I}\end{array}$ \\
\hline $\begin{array}{c}\text { MA } \\
\text { OD } \\
\text { V } \\
{[79]} \\
- \\
{[82]}\end{array}$ & $\begin{array}{c}\text { Rea } \\
\text { ctiv } \\
\mathrm{e}\end{array}$ & $\begin{array}{c}\text { Rec } \\
\text { eiv } \\
\text { er }\end{array}$ & $\begin{array}{c}\mathrm{H} \\
\text { ar } \\
\mathrm{d} \\
\mathrm{St} \\
\text { at } \\
\mathrm{e}\end{array}$ & $\begin{array}{l}\mathrm{N} \\
\mathrm{O}\end{array}$ & $\begin{array}{l}\mathrm{Y} \\
\mathrm{e} \\
\mathrm{s}\end{array}$ & $\begin{array}{c}\text { Lo } \\
\mathrm{w}\end{array}$ & $\begin{array}{l}\mathrm{N} \\
\mathrm{O}\end{array}$ & $\begin{array}{l}\mathrm{Y} \\
\mathrm{e} \\
\mathrm{s}\end{array}$ & $\begin{array}{l}\mathrm{N} \\
\mathrm{O}\end{array}$ & $\begin{array}{l}\mathrm{Y} \\
\text { es }\end{array}$ & $\begin{array}{l}\text { Y } \\
\text { e } \\
\text { s }\end{array}$ \\
\hline $\begin{array}{c}\text { AM } \\
\text { RIS } \\
{[98],} \\
{[99]}\end{array}$ & $\begin{array}{l}\text { Pro } \\
\text { acti } \\
\text { ve }\end{array}$ & $\begin{array}{l}\text { Sou } \\
\text { rce }\end{array}$ & $\begin{array}{c}\mathrm{H} \\
\text { ar } \\
\mathrm{d} \\
\mathrm{St} \\
\text { at } \\
\mathrm{e}\end{array}$ & $\begin{array}{l}\mathrm{N} \\
\mathrm{O}\end{array}$ & $\begin{array}{l}\mathrm{N} \\
\mathrm{O}\end{array}$ & $\begin{array}{c}\text { Lo } \\
\mathrm{w}\end{array}$ & $\begin{array}{l}\mathrm{Y} \\
\text { es }\end{array}$ & $\begin{array}{l}\text { Y } \\
\text { e } \\
\text { s }\end{array}$ & $\begin{array}{l}\mathrm{N} \\
\mathrm{o}\end{array}$ & $\begin{array}{l}\mathrm{Y} \\
\text { es }\end{array}$ & $\begin{array}{l}\text { Y } \\
\text { e } \\
\text { s }\end{array}$ \\
\hline $\begin{array}{c}\text { LA } \\
\text { M } \\
{[103} \\
], \\
{[104} \\
]\end{array}$ & $\begin{array}{c}\text { Rea } \\
\text { ctiv } \\
\text { e }\end{array}$ & $\begin{array}{l}\text { Sou } \\
\text { rce }\end{array}$ & $\begin{array}{c}\mathrm{S} \\
\text { of } \\
\mathrm{t} \\
\mathrm{St} \\
\text { at } \\
\mathrm{e}\end{array}$ & $\begin{array}{l}\mathrm{N} \\
\mathrm{o}\end{array}$ & $\begin{array}{l}\text { Y } \\
\text { e } \\
\text { s }\end{array}$ & $\begin{array}{l}\mathrm{Hi} \\
\text { gh }\end{array}$ & $\begin{array}{l}\mathrm{N} \\
\mathrm{O}\end{array}$ & $\begin{array}{l}\mathrm{N} \\
\mathrm{O}\end{array}$ & $\begin{array}{l}\mathrm{N} \\
\mathrm{O}\end{array}$ & $\begin{array}{l}\mathrm{N} \\
\mathrm{o}\end{array}$ & $\begin{array}{l}\text { Y } \\
\text { e } \\
\text { s }\end{array}$ \\
\hline $\begin{array}{c}\text { LGT } \\
{[87]}\end{array}$ & $\begin{array}{c}\text { Rea } \\
\text { ctiv } \\
\text { e }\end{array}$ & $\begin{array}{l}\text { Sou } \\
\text { rce }\end{array}$ & $\begin{array}{c}\mathrm{S} \\
\text { of } \\
\mathrm{t} \\
\mathrm{St} \\
\text { at } \\
\mathrm{e}\end{array}$ & $\begin{array}{l}\mathrm{N} \\
\mathrm{O}\end{array}$ & $\begin{array}{l}\mathrm{Y} \\
\mathrm{e} \\
\mathrm{s}\end{array}$ & $\begin{array}{l}\mathrm{Hi} \\
\text { gh }\end{array}$ & $\begin{array}{l}\mathrm{N} \\
\mathrm{O}\end{array}$ & $\begin{array}{l}\mathrm{N} \\
\mathrm{O}\end{array}$ & $\begin{array}{l}\mathrm{N} \\
\mathrm{O}\end{array}$ & $\begin{array}{l}\mathrm{N} \\
\mathrm{O}\end{array}$ & $\begin{array}{l}\mathrm{Y} \\
\mathrm{e} \\
\mathrm{s}\end{array}$ \\
\hline $\begin{array}{c}\text { BE } \\
\text { MR } \\
\text { P } \\
{[88],} \\
{[89]}\end{array}$ & $\begin{array}{l}\text { Pro } \\
\text { acti } \\
\text { ve }\end{array}$ & $\begin{array}{l}\text { Sou } \\
\text { rce }\end{array}$ & $\begin{array}{c}\mathrm{H} \\
\text { ar } \\
\mathrm{d} \\
\mathrm{St} \\
\text { at } \\
\mathrm{e}\end{array}$ & $\begin{array}{l}\mathrm{Y} \\
\mathrm{e} \\
\mathrm{s}\end{array}$ & $\begin{array}{l}\mathrm{N} \\
\mathrm{o}\end{array}$ & $\begin{array}{c}\text { Lo } \\
\mathrm{w}\end{array}$ & $\begin{array}{l}\mathrm{Y} \\
\text { es }\end{array}$ & $\begin{array}{l}Y \\
\text { e } \\
\text { s }\end{array}$ & $\begin{array}{l}\mathrm{N} \\
\mathrm{O}\end{array}$ & $\begin{array}{l}\mathrm{Y} \\
\text { es }\end{array}$ & $\begin{array}{l}\mathrm{N} \\
\mathrm{O}\end{array}$ \\
\hline $\begin{array}{c}\text { DD } \\
\mathbf{M} \\
{[83],} \\
{[84]}\end{array}$ & $\begin{array}{l}\text { Hy } \\
\text { brid }\end{array}$ & $\begin{array}{l}\text { Rec } \\
\text { eiv } \\
\text { er }\end{array}$ & $\begin{array}{c}\mathrm{S} \\
\text { of } \\
\mathrm{t} \\
\mathrm{St} \\
\text { at } \\
\mathrm{e}\end{array}$ & $\begin{array}{l}\mathrm{N} \\
\mathrm{o}\end{array}$ & $\begin{array}{l}\mathrm{N} \\
\mathrm{o}\end{array}$ & $\begin{array}{c}\text { Lo } \\
\mathrm{w}\end{array}$ & $\begin{array}{l}\mathrm{N} \\
\mathrm{O}\end{array}$ & $\begin{array}{l}\mathrm{Y} \\
\mathrm{e} \\
\mathrm{s}\end{array}$ & $\begin{array}{l}\mathrm{N} \\
\mathrm{O}\end{array}$ & $\begin{array}{l}\mathrm{Y} \\
\mathrm{es}\end{array}$ & $\begin{array}{l}\text { Y } \\
\text { e } \\
\text { s }\end{array}$ \\
\hline $\begin{array}{c}\text { STA } \\
\text { MP } \\
{[105} \\
]\end{array}$ & $\begin{array}{c}\text { Rea } \\
\text { ctiv } \\
\text { e }\end{array}$ & $\begin{array}{c}\text { Rec } \\
\text { eiv } \\
\text { er }\end{array}$ & $\begin{array}{c}\mathrm{H} \\
\text { ar } \\
\mathrm{d} \\
\mathrm{St} \\
\text { at } \\
\mathrm{e}\end{array}$ & $\begin{array}{l}\mathrm{N} \\
\mathrm{o}\end{array}$ & $\begin{array}{l}\mathrm{Y} \\
\mathrm{e} \\
\mathrm{s}\end{array}$ & $\begin{array}{c}\text { Lo } \\
\mathrm{w}\end{array}$ & $\begin{array}{l}\mathrm{N} \\
\mathrm{O}\end{array}$ & $\begin{array}{l}\mathrm{N} \\
\mathrm{O}\end{array}$ & $\begin{array}{l}\mathrm{N} \\
\mathrm{O}\end{array}$ & $\begin{array}{l}\mathrm{Y} \\
\text { es }\end{array}$ & $\begin{array}{l}\mathrm{N} \\
\mathrm{o}\end{array}$ \\
\hline
\end{tabular}




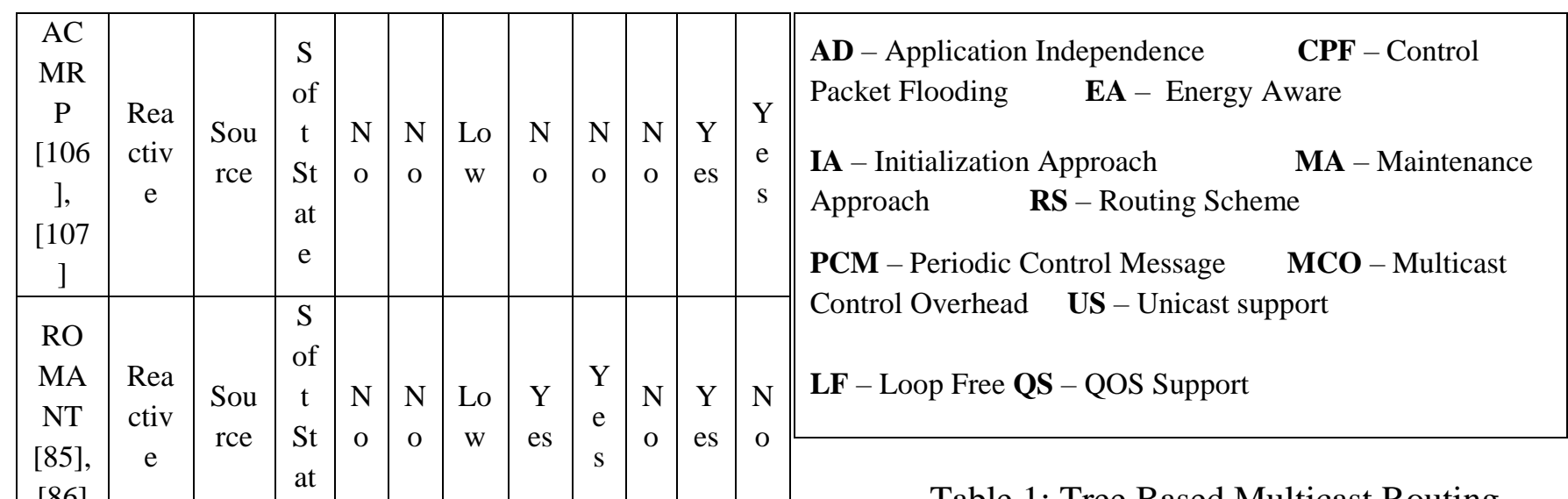

Table 1: Tree Based Multicast Routing Protocol

\begin{tabular}{|c|c|c|c|c|c|c|c|c|c|c|c|}
\hline PM & & & $\mathrm{H}$ & & & & & & & & \\
\hline RP & Pro & Soll & ar & $\mathrm{Y}$ & $\mathrm{N}$ & $\mathrm{Hi}$ & V & Y & & & \\
\hline 1 & acti & rce & St & $\mathrm{e}$ & 0 & $\mathrm{gh}$ & es & $\mathrm{e}$ & 0 & 0 & 0 \\
\hline [109 & ve & & at & S & & & & S & & & \\
\hline ] & & & $\mathrm{e}$ & & & & & & & & \\
\hline
\end{tabular}

\begin{tabular}{|c|c|c|c|c|c|c|c|c|c|c|c|}
\hline $\begin{array}{c}\text { Protocol } \\
\text { Name }\end{array}$ & RS & IA & MA & EA & US & MCO & PCM & $\mathbf{L F}$ & OS & CPF & AI \\
\hline $\begin{array}{l}\text { ODMRP } \\
{[95],[96]}\end{array}$ & Proactive & Source & $\begin{array}{l}\text { Soft } \\
\text { State }\end{array}$ & No & No & Low & Yes & Yes & No & Yes & Yes \\
\hline $\begin{array}{c}\text { CAMP [91], } \\
\text { [92] }\end{array}$ & Proactive & Receiver & $\begin{array}{l}\text { Soft } \\
\text { State }\end{array}$ & No & No & High & No & Yes & No & No & Yes \\
\hline $\begin{array}{c}\text { DCMP [93], } \\
\text { [94] }\end{array}$ & Reactive & Source & $\begin{array}{l}\text { Soft } \\
\text { State }\end{array}$ & No & No & High & No & Yes & No & Yes & Yes \\
\hline $\begin{array}{c}\text { FGMP } \\
{[110]}\end{array}$ & Reactive & Source & $\begin{array}{l}\text { Hard } \\
\text { State }\end{array}$ & No & Yes & Low & No & Yes & No & Yes & No \\
\hline $\begin{array}{c}\text { NSMP } \\
{[111]}\end{array}$ & Reactive & Source & $\begin{array}{l}\text { Soft } \\
\text { State }\end{array}$ & No & No & Low & Yes & Yes & No & Yes & No \\
\hline $\begin{array}{c}\text { E-ODMRP } \\
\text { [97] }\end{array}$ & Proactive & Receiver & $\begin{array}{l}\text { Soft } \\
\text { State }\end{array}$ & No & No & Low & Yes & Yes & No & Yes & Yes \\
\hline $\begin{array}{c}\text { CQMP } \\
\text { [113] }\end{array}$ & Reactive & Source & $\begin{array}{l}\text { Hard } \\
\text { State }\end{array}$ & No & No & High & No & Yes & No & No & No \\
\hline SRMP [112] & Reactive & Source & $\begin{array}{l}\text { Soft } \\
\text { State }\end{array}$ & No & No & Low & No & Yes & Yes & No & No \\
\hline
\end{tabular}




\begin{tabular}{|l|c|c|c|c|c|c|c|c|c|c|c|}
\hline BODS [114] & Reactive & Receiver & $\begin{array}{c}\text { Hard } \\
\text { State }\end{array}$ & No & No & High & No & Yes & No & No & Yes \\
\hline $\begin{array}{c}\text { MPGC } \\
{[115]}\end{array}$ & Proactive & Source & $\begin{array}{c}\text { Soft } \\
\text { State }\end{array}$ & Yes & No & Low & Yes & Yes & No & Yes & No \\
\hline AD - Application Independence & CPF - Control Packet Flooding $\quad$ EA - Energy Aware \\
IA - Initialization Approach \\
MA - Maintenance Approach $\quad$ RS - Routing Scheme \\
PCM - Periodic Control Message $\quad$ MCO - Multicast Control Overhead US - Unicast support \\
LF - Loop Free QS - QOS Support
\end{tabular}

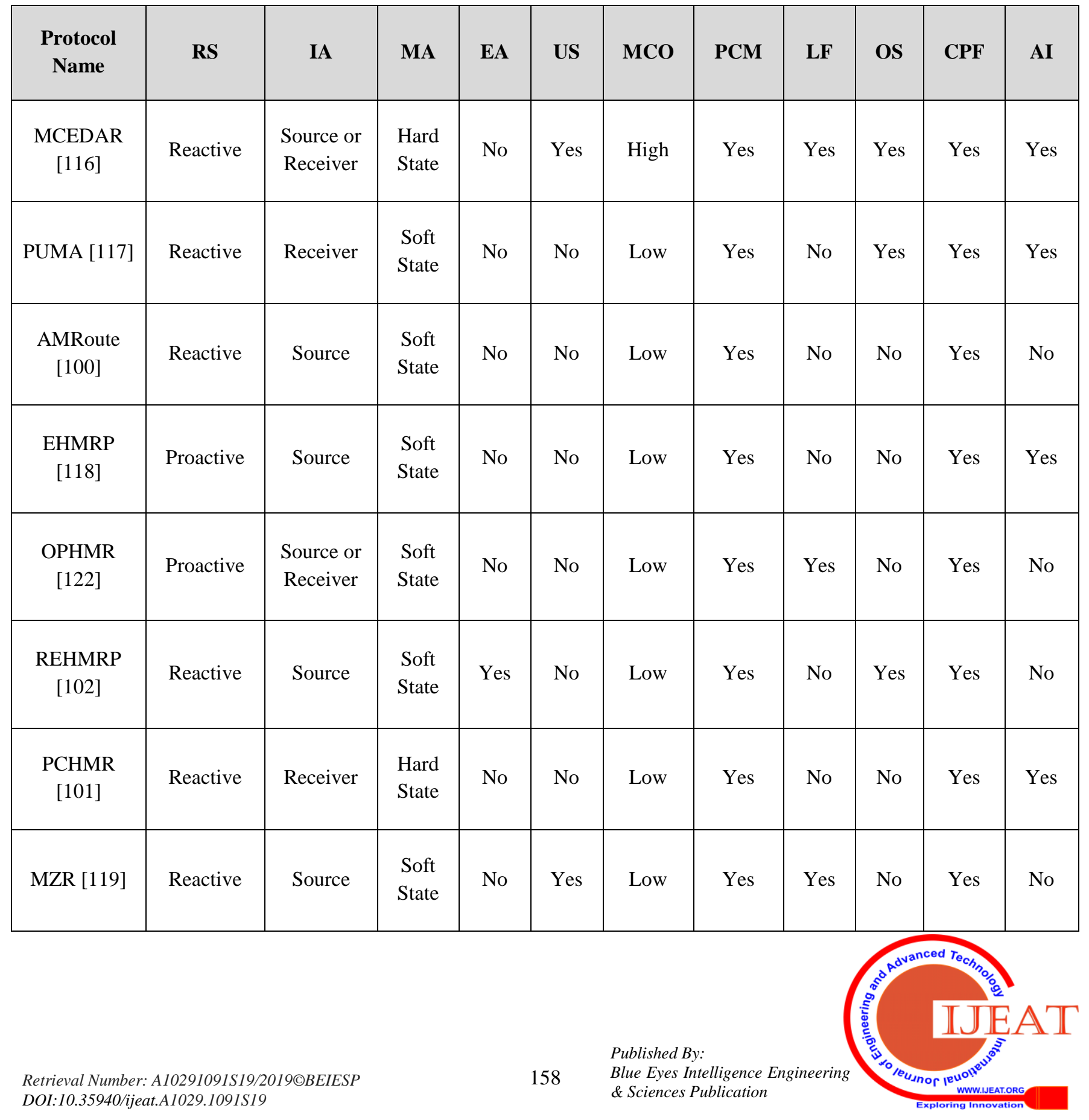




\begin{tabular}{|c|c|c|c|c|c|c|c|c|c|c|c|}
\hline $\begin{array}{c}\text { MAMR } \\
{[120]}\end{array}$ & Reactive & Source & $\begin{array}{l}\text { Soft } \\
\text { State }\end{array}$ & No & No & Low & Yes & Yes & Yes & Yes & No \\
\hline HVDB [121] & Proactive & $\begin{array}{l}\text { Source or } \\
\text { Receiver }\end{array}$ & $\begin{array}{l}\text { Hard } \\
\text { State }\end{array}$ & No & No & Low & Yes & Yes & Yes & Yes & No \\
\hline \multicolumn{7}{|c|}{ AD - Application Independence } & \multicolumn{5}{|c|}{ EA - Energy Aware } \\
\hline \multicolumn{3}{|c|}{ IA - Initialization Approach } & \multicolumn{4}{|c|}{ MA-Maintenance Approach } & \multicolumn{5}{|c|}{ RS - Routing Scheme } \\
\hline \multicolumn{3}{|c|}{ PCM - Periodic Control Message } & \multicolumn{4}{|c|}{ MCO - Multicast Control Overhead } & \multicolumn{3}{|c|}{ US - Unicast support } & & \\
\hline
\end{tabular}

Table 3: Hybrid Based Multicast Routing Protocol

\section{REFERENCE}

1. Divya M, Dr S Subasree, and Dr N K Sakthivel, "Performance Analysis of Efficient Energy Routing Protocols in MANET", Procedia Computer Science, Year: 2015, Volume: 57, Pages: 890-897.

2. AndyAn-Kai Jeng, and Rong-Hong Jan, "Adaptive Topology Control for Mobile Ad Hoc Networks", and IEEE Transactions on Parallel and Distributed Systems, Year: 2011, Volume: 22, Issue: 12, Pages: 1953 - 1960.

3. Xiaojiang Du, Dapeng Wu, Wei Liu, and Yuguang Fang, "Multiclass routing and medium access control for heterogeneous mobile ad hoc networks", IEEE Transactions on Vehicular Technology, Year: 2006, Volume: 55, Issue: 1 Pages: 270 - 277.

4. Shivashankar, Hosahalli Narayanagowda Suresh, Golla Varaprasad, and Guruswamy Jayanthi," Designing Energy Routing Protocol With Power Consumption Optimization in MANET " in IEEE Transactions On Emerging Topics In Computing, Year: 2014, Volume: 2, Issue: 2, Pages: 192 - 197.

5. P.J. Wan, G. Calinescu, X.-Y.Li, and O.Frieder, "Minimum-energy broadcast routing in static ad hoc wireless networks", IEEE INFOCOM 2001, Year: 2001, Volume: 2, Pages: 1162 - 1171.

6. S. Chakrabarti, and A. Mishra, "QoS issues in ad hoc wireless networks", IEEE Communications Magazine, Year: 2001, Volume: 39, Issue: 2, Pages: 142 - 148.

7. D. Helen and D. Arivazhagan, "Applications, Advantages and Challenges of Ad Hoc Networks", Journal of Academia and Industrial Research (JAIR), Year: 2014, Volume: 2, Issue: 8, Pages: 453 - 457.

8. Siddhu Warrier, "Characterisation and Applications of MANET Routing Algorithms in Wireless Sensor Networks", Master of Science School of Informatics, University of Edinburgh 2007.

9. Naeem Raza, Muhammad Umar Aftab, Muhammad Qasim Akbar, Omair Ashraf, Muhammad Irfan, "Mobile Ad-Hoc Networks Applications and Its Challenges", Scientific Research Publishing
Communications and Network, Year :2016, volume: 8, pages: $131-136$.

10. E.M. Royer, Chai-Keong Toh, "A review of current routing protocols for ad hoc mobile wireless networks", IEEE Personal Communications, Year: 1999, Volume: 6, Issue: 2, Pages: 46 - 55.

11. B. Kannhavong, H. Nakayama, Y. Nemoto, N. Kato and A. Jamalipour, "A survey of routing attacks in mobile ad hoc networks", IEEE Wireless Communications, Year: 20017, Volume: 14, Issue: 5.pages: $85-91$.

12. Aniket Deshpande," Significance of Mobile Ad-hoc Networks in the Growth of Cloud Based Services", International Journal of Computer and Communication Engineering, Year: 2016, Volume: 5, Issue: 3, Pages: $222-228$

13. Prabhleen Kaur, and Sukhman, "An Overview on MANET- Advantages, Characteristics and Security Attacks", International Journal of Computer Applications, ICAET 2016, Pages: 0975 - 8887.

14. Diaa Eldein Mustafa Ahmed, Othman O. Khalifa, “An Overview of MANETs: Applications, Characteristics, Challenges and Recent Issues", International Journal of Engineering and Advanced Technology (IJEAT), Year: 2017, Volume: 6, Issue: 4, Pages: 128-133.

15. Andrea Goldsmith," WIRELESS COMMUNICATIONS", Cambridge university press, 2007.

16. Satyabrata Chakrabarti, Amitabh Mishra," QoS Issues in Ad Hoc Wireless Networks", IEEE Communications Magazine, Year: 2001, Volume: 39, Issue: 2, Pages: $142-148$.

17. Pattnaik, Prasant Kumar, Mall, Rajib, "Fundamentals of Mobile Computing", Second Edition, PHI Learning Pvt. Ltd., 2015.

18. Atsushi Yoshinari, Hiroki Nishiyama, Nei Kato, Dan Keun Sung, "Dynamic topology update mechanism in local tree-

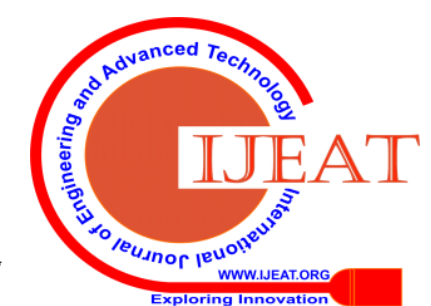


based reliable topology (LTRT) based MANETs", IEEE Conference Publications, Year: 2012, Pages: $281-285$

19. Sonika Kandari, M. K. Pandey, "Evaluation of energy consumption by nodes of MANE T", IEEE Conference Publications, Year: 2015, Pages: $32-35$

20. Stefan Stieglitza and Christoph Fuch, "Challenges of MANET for Mobile Social Networks", Procedia Computer Science, Year: 2011, Volume: 5, Pages 820 825.

21. Saad M.AdamRosilahHassan; "Delay aware Reactive Routing Protocols for QoS in MANETs: a Review", published by science direct, Journal of Applied Research and Technology, Year: 2013, Volume: 11, Issue: 6, Pages 844-850.

22. Ch. Niranjan Kumar, N. Satyanarayana, "Multipath QoS Routing for Traffic Splitting in MANETs", published by science direct and Procedia Computer Science, Year: 2015, Volume: 48, Pages 414-426.

23. $\mathrm{X}$. Chen and J. Wu, "Multicasting techniques in mobile ad-hoc networks", ACM transaction, Year: 2003, Pages: 25-40.

24. Zhi Ren , Wei Guo," Unicast routing in mobile ad hoc networks: present and future directions", IEEE conference publication, Year: 340-344, 2003.

25. Hadi Sargolzaey, Ayyoub Akbari Moghanjoughi and Sabira Khatun," A Review and Comparison of Reliable Unicast Routing Protocols For Mobile Ad Hoc Networks", IJCSNS, Year: 2009, Volume: 9, Issue: 1, Pages: 186-196.

26. Kamal Kant and Lalit K. Awasthi, "Unicast and Multicast Routing Protocols for Manets: A Comparative Survey", Year: 2010.

27. Natarajan Meghanathan,"A Unicast MANET Routing Protocol to Simultaneously Minimize the Stability-Hop Count Tradeoff and End-to-End Delay", IEEE conference publication, Year: 2012, Pages: 60 - 64 .

28. Wang Ke, Ou Yangrui, Ji Hong, Zhang Heli, and Li $\mathrm{Xi}$, "Energy aware hierarchical cluster-based routing protocol for WSNs", The Journal of China Universities of Posts and Telecommunications, Year: 2016, Volume: 23, Issue: 4, Pages 46-52.

29. Xuxun Liu," A typical Hierarchical Routing Protocols for Wireless Sensor Networks: A Review", IEEE Sensors Journal Year: 2015, Volume: 15, Issue: 10, Pages: $5372-5383$.

30. DaWei $\mathrm{Xu}$, Jing Gao, "Comparison Study to Hierarchical Routing Protocols in Wireless Sensor Networks", Procedia Environmental Sciences, Year: 2011, Volume: 10, Pages 595-600.

31. Karan Soodl and Nagendra sah, "New Unicast Routing Protocol Using Comparative Study of Proactive, Reactive and Hybrid protocols for MANET", International Journal of Engineering Research and General Science, Year: 2014 Volume: 2, Issue: 4, Pages: $44-50$.

32. M.Sravan Kumar Reddy and Vikram Narayandas, "A Classification of various unicast and multicast Routing protocols in MANET", International Journal of Engineering and Computer Science, Year: 2015, Volume: 4, Issue: 4, Page No. 11571-11581.

33. Hamza Toulni and Benayad Nsiri, "Hybrid Routing Protocol for VANET Using Ontology", Procedia
Computer Science, Year: 2015, Volume: 73, Pages: 94-101.

34. Christian Maihofer, "A survey of geocast routing protocols", IEEE Communications Surveys \& Tutorials, Year: 2004, Volume: 6, Issue: 2 Pages: $32-42$.

35. S. Doi; S. Ata; H. Kitamura; M. Murata,"IPv6 anycast for simple and effective service-oriented communications", IEEE Communications Magazine, Year: 2004, Volume: 42, Issue: 5, Pages: 163 - 171.

36. Ling Song; Zheng-bing Xia, "An Anycast Routing Protocol for Wireless Mesh Access Network", IEEE Conference Publications, WASE International Conference on Information Engineering, Year: 2009, Volume: 2, Pages: 82 - 85.

37. Dong Xuan; Weijia Jia; Wei Zhao; Hongwen Zhu, "A routing protocol for anycast messages", IEEE Transactions on Parallel and Distributed Systems, Year: 2000, Volume: 11, Issue: 6, Pages: 571 - 588.

38. Vesal Hakami and Mehdi Dehghan, "Efficient broadcasting in slow fading wireless ad-hoc networks", Journal of Network and Computer Applications, Year: 2014, Volume 46, Pages 113-123.

39. Abhishek Sinha, Georgios Paschos and Eytan Modiano,"Throughput-Optimal Multi-Hop Broadcast Algorithms", IEEE/ACM Transactions on Networking, Year: 2017, Volume: 25, Issue: 5, Pages: 3088 - 3101 .

40. D.G.Reinaa and M.GünesbS.L.Torala, "Real experimentation of probabilistic broadcasting algorithms based on dissimilarity metrics for multi-hop ad hoc networks", Elsevier publication on Ad Hoc Networks, Year: 2016, Volume: 47, Issue: 1, Pages 115.

41. Fei Dai and Jie Wu," Performance analysis of broadcast protocols in ad hoc networks based on self-pruning", IEEE Transactions on Parallel and Distributed Systems, Year: 2004, Volume: 15, Issue: 11, Pages: 1027 - 1040.

42. Majid Khabbazian, Ian F. Blake, and Vijay K. Bhargava, "Local Broadcast Algorithms in Wireless Ad Hoc Networks: Reducing the Number of Transmissions", IEEE Transactions On Mobile Computing, Year: 2012, Volume. 11, Issue: 3, Pages: $402-413$.

43. Koushik Sinha, Suranjan Ghose, and Pradip K. Srimani, "Fast deterministic broadcast and gossiping algorithms for mobile ad hoc networks", Journal of Parallel and Distributed Computing, Year: 2008, Volume: 68, Issue: 7, Pages 922-938.

44. Yijin Zhang; Kenneth W. Shum; Wing Shing Wong; Feng Shu, "An energy-aware reliable deterministic broadcast protocol for wireless sensor networks" IEEE Conference Publications (GC Wkshps), Year: 2014, Pages: $1427-1432$.

45. Wilson Woon; Kwan L. Yeung, "Enhanced Termination Condition for Deterministic

Broadcasting Protocols in Mobile Ad Hoc Networks", IEEE 72nd Vehicular Technology Conference, Year: 2010, Pages: $1-5$.

46. Shafinaz Buruhanudeen; Mohamed Othman; Mazliza Othman; Borhanuddin Mohd Ali, "Mobility models, broadcasting methods and factors contributing towards the efficiency of the MANET routing protocols: Overview", IEEE International Conference on Telecommunications 
and Malaysia International Conference on Communications, Year: 2007 Pages: 226 - 230.

47. T. Pongthawornkamol, K. Nahrstedt, and G. Wang, "HybridCast: A Hybrid Probabilistic/Deterministic Approach for Adjustable Broadcast Reliability in Mobile Wireless Ad Hoc Networks", IEEE International Conference on Communications, Year: 2009 Pages: 1 - 6.

48. Minglu Li; Ling Ding; Yifeng Shao; Zhensheng Zhang; Bo Li, "On Reducing Broadcast Transmission Cost and Redundancy in Ad Hoc Wireless Networks Using Directional Antennas", IEEE Transactions on Vehicular Technology, Year: 2010, Volume: 59, Issue: 3, Pages: $1433-1442$.

49. Xu Li; Tianjiao Liu; Ying Liu; Yan Tang, "Optimized multicast routing algorithm based on tree structure in MANETs", IEEE Transactions, China Communications, Year: 2014 Volume: 11, Issue: 2, Pages: 90 - 99.

50. Abdulmalek Al-hemyari; Kasmiran Jumari; Mahamod Ismail; Sabri Saeed, "A comparative survey of multicast routing protocol in MANETs", IEEE Conference Publications, ICCIS, Year 2009, Volume: 2, Pages: 830 - 835.

51. Anupama Sharma, Abhay Bansal and Vinay Rishiwal, "Assessment of QoS based multicast routing", Pages: $421-426$.

52. Mahdi Ghasemi and Mozafar Bag-Mohammady, "Classification of multicast routing protocols for Mobile Ad Hoc Networks", IEEE Conference Publications, International Conference on ICT Convergence (ICTC), Year: 2012, Pages: 789 - 794.

53. Bala Krishna Maddali,"Core network supported multicast routing protocol for wireless sensor networks", IET Wireless Sensor Systems, Year: 2015, Volume: 5, Issue: 4.

54. Ajay Kumar Yadav and Sachin Tripathi, "Design of efficient multicast routing protocol using limited flooding mechanism", IEEE Conference Publications, Micro-Com, Year: 2016, Pages: 1 - 6.

55. Xiao Chen and Jie $\mathrm{Wu}$, "Multicasting Techniques in Mobile Ad Hoc Networks", CRC Press LLC, 2003.

56. C. Siva Ram Murthy and B. S. Manoj, "Ad Hoc Wireless Networks Architectures and Protocols", Pearson Education, 2004.

57. Subir Kumar Sarkar, T.G. Basavaraju and C. Puttamadappa, "Ad Hoc Mobile Wireless Networks: Principles, Protocols and Applications", CRC Press, 2013.

58. Shafiullah Khan, Al-Sakib Khan Pathan and Nabil Ali Alrajeh, "Wireless Sensor Networks: Current Status and Future Trends", CRC Press, 2016.

59. L. Junhai, X. Liu and Y. Danxia, "Research on multicast routing protocols for mobile ad-hoc networks", Computer Networks, Year: 2008, Volume: 52, Issue: 5, Pages 988-997.

60. M. R. BAKER and M. A. AKCAYOL, "A Survey of Multicast Routing Protocols in Ad-Hoc Networks", Gazi University Journal of Science GU J Sci, Year: 2011, Volume: 24, Pages: 451-462.

61. Haojun Huang, Junbao Zhang. Xu Zhang, Benshun Yi, and Feng Li, "EMGR: Energy-efficient multicast geographic routing in wireless sensor networks",
Elsevier journal publication on computer networks, Year: 2017, Volume: 129, Pages: 51-63.

62. Gyanappa A. Walikar and Rajashekar C. Biradar, "Energy aware multicast routing in mobile ad-hoc networks using NS-2”, IEEE International Conference on Electrical, Computer and Communication Technologies (ICECCT), Year: 2015, Pages: 1 - 7.

63. $\mathrm{Xu} \mathrm{Li,} \mathrm{Tianjiao} \mathrm{Liu,} \mathrm{Ying} \mathrm{Liu,} \mathrm{andYan} \mathrm{Tang,}$ "Optimized multicast routing algorithm based on tree structure in MANETs", IEEE Journals \& Magazines, China Communications, Year: 2014, Volume: 11, Issue: 2, Pages: $90-99$.

64. Tsung-Chuan Huang, Chien-Te Wu and Hung-Yi Chi, "Robust Mesh-based Multicast Protocol with a secondroute discovery scheme in MANET", IET 2nd International Conference on Wireless, Mobile and Multimedia Networks, Year: 2008, Pages: 414 - 417

65. Guangmin Hu; R. K. C. Chang, "Forwarding state scalability-aware multicast routing", 10th IEEE Symposium on Computers and Communications, Year: 2005, Pages: 395 - 400.

66. Vishal Nagar; Yashpal Singh; D. C. Dhubkarya, "Estimation of Reliability and Scalability in Ad-Hoc Multicast Routing Protocol for Sender Network", IEEE Conference Publications, International Conference on Computational Intelligence and Communication Networks Year: 2011, Pages: 350 - 353.

67. Jing Dong, Reza Curtmola Cristina and Nita-Rotaru, "Secure High-Throughput Multicast Routing in Wireless Mesh Networks", IEEE Transactions on Mobile Computing, Year: 2011, Volume: 10, Issue: 5, Pages: 653 - 668 .

68. A. Menaka Pushpa; K. Kathiravan, "Secure multicast routing protocol against internal attacks in mobile ad hoc networks", IEEE GCC Conference and Exhibition, Year: 2013, Pages: 245 - 250.

69. Reza Curtmola, Student Member,Cristina NitaRotaru,and Senior Member, "BSMR: ByzantineResilient Secure Multicast Routing in Multihop Wireless Networks", IEEE TRANSACTIONS ON MOBILE COMPUTING, Year: 2009, Volume: 8, Issue: 4, Pages: $445-459$.

70. Natarajan Meghanathan, “A MANET Multicast Routing Protocol for Stable Trees based on the Inverse of Link Expiration Times", IEEE Consumer Communications and Networking Conference, Year: 2012, Pages: 947 - 951.

71. Koushik Sinha, Bidyut Gupta, Shahram Rahimi and Ansuman Bhattacharya, "Candidate Core Selection for Load-Balanced Multicore Shared Tree Multicasting", 3rd International Conference on Recent Advances in Information Technology (RAIT), Year: 2016, Pages: 35 $-40$.

72. Rajneesh Gujral, Sanjeev Rana and Amrita Chaudhary, "Study and Comparison of Mesh and Tree- Based Multicast Routing Protocols for MANETs", International Journal of Latest Trends in Engineering and Technology, Year: 2012, Volume: 1, Issue: 2, Pages: 28 - 42.

73. G.S.Sreedhar and Dr.A.Damodaram, "Tree Based Multicast Routing Protocols for Mobile Ad hoc Networks and Current State of the Art", International Journal of

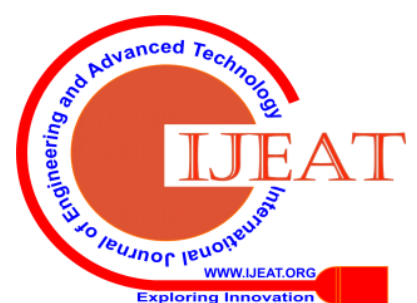


Science and Advanced Technology, Year: 2011, Volume: 1, Issue: 10, Pages: 37 - 42.

74. Sagar P. Latake, Gitanjali R. Shinde, and Rajesh H. Kulkarni," Tree, Mesh Structure Based and Stateless Multicast Routing Protocols in Wireless Networks", (IJCSIT) International Journal of Computer Science and Information Technologies, Year: 2013, Volume: 4, Issue: 3, Pages: 485-488.

75. Radhika Ranjan Roy, "Handbook of Mobile Ad Hoc Networks for Mobility Models", Springer Science \& Business Media, Year: 2010.

76. Rajashekhar Biradar, Sunilkumar Manvi, Member, IACSIT, and Mylara Reddy," Mesh Based Multicast Routing in MANET: Stable Link Based Approach", International Journal of Computer and Electrical Engineering, Year: 2010, Volume: 2, Issue: 2, Pages: 371-380.

77. P. Prasanna Murali Krishna, Dr. M.V. Subramanyam and Dr. K.Satya Prasad, "Mesh Based and Hybrid Multicast Routing Protocols for Manets: Current State of the Art", Global Journal of Computer Science and Technology, Year: 2012, Volume: 12, Issue: 6, Pages: $13-22$.

78. Ashema Hasti and U. S. Pandey," Analysis of performance of multicasting routing protocol MAODV for QoS parameters using NS2", Computing for Sustainable Global Development (INDIACom), IEEE publications, Year: 2015, Pages: 845 - 848.

79. Mingyang Zhong, Yunqing $\mathrm{Fu}$ and Xinqiang Jia, "MAODV multicast routing protocol based on node mobility prediction", E-Business and E - Government (ICEE), IEEE publications, Year: 2011, Pages: 1 - 4.

80. Sun, Baolin, Li and Layuan, "QoS-aware multicast routing protocol for Ad hoc networks", Journal of Systems Engineering and Electronics Year: 2006, Volume: 17, Issue: 2, Pages: 417-422.

81. Jayalakshmi G Naragund and R M Banakar, "Multicast Routing in Scalable Networks using MAODV", International Journal of Advanced Computer Research, Year: 2013, Volume: 3, Issue: 11, Pages: 47 - 53.

82. Lusheng Ji and M.S. Corson, "Differential destination multicast-a MANET multicast routing protocol for small groups", Twentieth Conference of the IEEE Computer and Communications Societies, Year: 2001, Volume: 2, Pages: 1192 - 1201.

83. LUSHENG JI and M. SCOTT CORSON, "Explicit Multicasting for Mobile Ad Hoc Networks", Mobile Networks and Applications, 8, Year: 2003, Volume: 8, Pages: 535-549.

84. R. Vaishampayan; J.J. Garcia-Luna-Aceves, "Robust tree-based multicasting in ad hoc networks", IEEE International Conference on Performance, Computing, and Communications, Year: 2004, Pages: 647 - 652.

85. Eric Astier,Abdelhakim Hafid and Sultan Aljahdali, " An efficient mesh-based multicast routing protocol in mobile ad hoc networks", Journal on wireless communication and mobile computing, Year: 2012, Volume: 12, Issue: 9, Pages: 822-836.

86. Ma Xiang, "Analysis on Multicast Routing Protocols for Mobile Ad Hoc Networks", Physics Procedia, Year: 2012, Volume: 25, Pages: 1787 - 1793.

87. Juan A. Sanchez, Pedro M. Ruiz, Member, Jennifer Liu, and Ivan Stojmenovic, Member, "Bandwidth-Efficient Geographic Multicast Routing Protocol for Wireless
Sensor Networks", IEEE SENSORS JOURNAL, Year: 2007, Volume: 7, Issue: 5, Pages: 627 - 636.

88. Tomochika Ozaki, Jaime Bae Kim and Tatsuya Suda, "Bandwidth-Efficient Multicast Routing Protocol for Ad-Hoc Networks", IEEE INFOCOM, Year: 2001, Pages: $10-17$.

89. Arshta Kaur and Gurmeen Kaur, "Highlighting the efficient multicast routing algorithms in mobile ad-hoc network", ICICT, International, Year: 2016, Volume: 1, Pages: 1 - 6.

90. J.J. Garcia-Luna-Aceves and E.L. Madruga, "A multicast routing protocol for ad-hoc networks", IEEE publication, Year: 1999, Volume: 2, Pages: 784 - 792.

91. Ewerton L. Madruga and J.J. Garcia-luna-aceves, "Scalable Multicasting: The Core-Assisted Mesh Protocol", Mobile Networks and Applications, Year: 2001, Volume: 6, Pages: 151-165.

92. K. Das, B. S. Manoj, and C. Siva Ram Murthy, "A Dynamic Core based Multicast Routing Protocol for Ad-hoc Wireless Networks", Proceeding of MobiHoc $\underline{02}$.

93. $\underline{\text { S. Mangai }}$ A. Tamilarasi and C. Venkatesh, "Dynamic core multicast routing protocol implementation using ANT colony optimization in ad hoc wireless networks", IEEE Publication, ICCC, Year: 2008, Pages: 1 - 5.

94. Sung-Ju Lee, M. Gerla and Ching-Chuan Chiang, "Ondemand multicast routing protocol", Wireless Communications and Networking Conference, Year: 1999, Volume, Pages: 1298 - 1302.

95. M.Rajendiran and S.K.Srivatsa, "Stable Route Link in on-Demand Multicast Routing Protocol for Ad Hoc Networks", Procedia Engineering, Year: 2012, Volume: 38, Pages 1391-1398.

96. Soon Y. Oh, Joon-Sang Park and Mario Gerla, "EODMRP: Enhanced ODMRP with motion adaptive refresh", Journal of Parallel and Distributed Computing, Year: 2008, Volume: 68, Issue: 8, Pages: 1044-1053.

97. Sagar P. Latake, Gitanjali R. Shinde and Rajesh H. Kulkarni, Tree, Mesh Structure Based and Stateless Multicast Routing Protocols in Wireless Networks, (IJCSIT) International Journal of Computer Science and Information Technologies, Year: 2013, Volume: 4, Issue: 3, Pages: 485-488.

98. 2. C.W. $\mathrm{Wu}$ and Y.C. Tay,"AMRIS: a multicast protocol for ad hoc wireless networks", Military Communications Conference Proceedings, MILCOM, Year: 1999, Volume: 1, Pages: 25 - 29.

99. Bommaiah, McAuley and Talpade, "AMRoute: Adhoc Multicast Routing Protocol”, Internet Engineering Task Force, Internet-Draft, 1999.

100. Wei-Hsiang Cheng, Chung-Yi Wen and Kai-Ten Feng, "Power-Controlled Hybrid Multicast Routing Protocol for Mobile Ad Hoc Networks", IEEE Vehicular Technology Conference, Conference publication, Year: 2006, Volume: 3, Pages: 1087 - 1091.

101. Gyanappa A. Walikar and Rajashekhar C. Biradar, "Reliable and energy efficient hybrid multicast routing in Mobile Ad Hoc", International Conference on, IEEE Conference publication, Year: 2016, Pages: 1077 1083.

102. Carlos de Morais Cordeiro and Dharma Prakash 
Agrawal,"Ad Hoc and Sensor Networks: Theory and Applications", published by World Scientific Publishing Co Pvt Ltd, 2011.

103. R.Periasamy, C.Ranjithkumar and P.Panimalar, "A Study on Multicast Routing Protocols for MANETS: MRMP, ERAMOBILE, TSMP, LAM, PUMA"; IJCSNS International Journal of Computer Science and Network Security, Year: 2013, Volume: 13, Issue: 9, Pages: 93 - 97.

104. Lucile Canourgues, Jerome Lephay, Laurent Soyer and Andre-luc Beylot, "STAMP: Shared-Tree Ad Hoc Multicast Protocol", Military Communications Conference, IEEE Conference publication, Year: 2006, Pages: $1-7$.

105. Sangho Park And Daeyeon Park," Adaptive Core Multicast Routing Protocol", Kluwer Academic Publishers, Wireless Networks, Year: 2004, Volume: 10, Pages: 53-60.

106. B. Kaliaperumal, A. Ebenezer and Jeyakumar, "Adaptive Core Based Scalable Multicasting Networks", IEEE Conferences INDICON, Year: 2005, Pages: 198 - 202.

107. G. Song, V. Leung, and O. Yang, "A distributed minimum energy multicast algorithm in MANETs," in Proc. WoWMoM, Year: 2006, Pages: 134-140.

108. Nen-Chung Wang, Jong-Shin Chen, Yung-Fa Huang and $\mathrm{Yu}-\mathrm{Li} \mathrm{Su}$, "A Power-Aware Multicast Routing Protocol for Mobile Ad Hoc Networks With Mobility Prediction", Wireless Pers Commun Year: 2007, Volume: 43, Pages: 1479-1497.

109. Ching Chuan Chiang, Mario Gerla and Lixia Zhan, "Forwarding Group Multicast Protocol (FGMP) for multihop, mobile wireless networks", Springer publication on Cluster Computing, Year: 1998, Volume: 1, Issue: 2, Pages: 187-196.

110. Seungjoon Lee and Chongkwon Kim, "Neighbor supporting ad hoc multicast routing protocol", IEEE publication, Mobile and Ad Hoc Networking and Computing, Year: 2000, Pages: 37 - 44.

111. H. Labiod, H. Moustafa, "The Source Routing-based Multicast Protocol for Mobile Ad Hoc Networks (SRMP)", Internet draft, IETF, November 2001.

112. H. Dhillon and H.Q. Ngo, "CQMP: a mesh-based multicast routing protocol with consolidated query packets", Wireless Communications and Networking Conference, IEEE Publication, Year: 2005, Volume: 4, Pages: 2168 - 2174.

113. W.K.G. Seah, "Distributed Steiner-Like Multicast Path Setup for Mesh-based Multicast Routing in Ad Hoc Networks", IEEE International Conference on Sensor Networks, Year: 2006, Volume: 2, Pages: 192 - 197.

114. Y. Leu, M.H Tsai, and C. Tzu-Chiang, "Adaptive power aware clustering and multicasting protocol for mobile ad-hoc networks", UIC, Year: 2006, Page: 331340.

115. Sinha, R. Sivakumar and V. Bharghavan, "MCEDAR: multicast core-extraction distributed ad hoc routing", IEEE publication Wireless Communications and Networking Conference, Year: 1999, Volume: 3, Pages: 1313 - 1317.

116. A. Menaka Pushpa and K. Kathiravan, "Resilient PUMA (Protocol for Unified Multicasting through Announcement) against internal attacks in Mobile Ad hoc Networks", Year: 2013, Pages: 1906 - 1912.
117. J. Biswas, M. Barai and S.K. Nandy,"Efficient hybrid multicast routing protocol for ad-hoc wireless networks", 29th Annual IEEE International Conference on Local Computer Networks, Year: 2004, Pages: 180 187.

118. V. Devarapalli and D. Sidhu, "MZR: a multicast protocol for mobile ad hoc networks", IEEE International Conference on Communications, Year: 2001, Volume: 3, Pages: 886 - 891.

119. H. M. P. Shekhar, M. A. Arun Kumar, and K. S. Ramanatha, "Mobile agents aided multicast routing in mobile ad-hoc networks", IEEE ICACT, Year: 2005, Volume: 2, Page: 765-770.

120. G. Wang, C. Guojun, and L. Zhang, et al., "A novel QoS multicast model in mobile ad-hoc networks", IEEE-IPDPS, Year: 2005, Pages: 206b - 206b.

121. Adel Ben Mnaouer, Lei Chen, Chuan Heng Foh and Juki Wirawan Tantra, " OPHMR: An Optimized Polymorphic Hybrid Multicast Routing Protocol for MANET", IEEE Transactions on Mobile Computing, Year: 2007, Volume: 6, Issue: 5, Pages: 551 - 562.

122. Tracy Camp, and Yu Liu, "An adaptive mesh-based protocol for geocast routing", Journal of Parallel Distributed Computing, Year: 2003, Volume: 63, Pages:196-213.

\section{AUTHORS PROFILE}

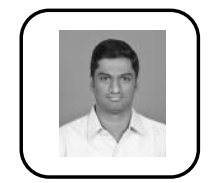

V.Sabaresan received the B.Tech and M.Tech degrees from Anna University, Tamilnadu, India. In 2012, he joined the Department of computer science in Kalaivani College of technology, as an assistant professor. Since December 2016, he has been with the Department of Information Technology, Agni College of Technology, where he was an Assistant Professor. His current research interests include machine learning intelligent, Adhoc and body sensor Networks.

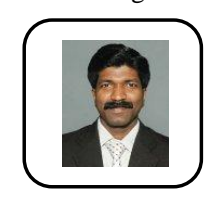

S.Godfrey Winster received the $\mathrm{PhD}$ form Anna University and he has 15 years of teaching experience in various reputed engineering colleges in Tamilnadu, India. Since June 2013, he has been with the Department Computer Science and Engineering, Saveetha Engineering College, where he was a professor. His current research interests include Web Mining, Semantic Web, Cloud Computing, Data Analytics and Social Networking. 